\title{
An exactly solvable multiple stochastic optimal stopping problem
}

\section{Hidekazu Yoshioka ${ }^{*}$}

\section{"Correspondence:}

yoshih@life.shimane-u.ac.jp

${ }^{1}$ Faculty of Life and Environmental

Science, Shimane University,

Matsue, Japan

\section{Springer}

\begin{abstract}
A new kind of multiple stochastic optimal stopping problem is formulated and its associated recursive variational inequalities are derived. We show that these variational inequalities can be solved exactly in a cascading manner. The relevance of the present problem in analyzing animal migration, which is an ecologically important problem, is also briefly discussed.
\end{abstract}

Keywords: Multiple optimal stopping; Geometric Brownian motion; Dynamic programming principle; Variational inequality

\section{Introduction}

Stochastic optimal stopping models are useful mathematical tools for analyzing decisionmaking processes in the fields of financial [1, 2], environment [3, 4], and ecology [5-7]. Multiple optimal stopping problems based on stochastic differential equations (SDEs) are among the ones that have been analyzed most in detail because of their rich mathematical structures $[8,9]$. Exactly solvable multiple optimal stopping models are useful from both theoretical and practical point of views $[10,11]$.

We are interested in solvability of a multiple optimal stopping problem that has not been focused on so far, which is related to animal migration: an important ecological problem. Our problem is explained below. In this paper, the summation $\sum_{i=j}^{k} a_{i}$ of a sequence $a_{i}$ is replaced by 0 when $k<j$. Let $B_{t}$ for $t \geq 0$ be a standard 1-D Brownian motion on the probability space as in the usual setting [12]. Its associated completed filtration is denoted by $F=\left\{F_{t}\right\}_{t>0}$. Let $\tau_{0}=0$. We consider a multiple optimal stopping problem of finding the collection of the stopping times $\tau=\left(\tau_{1}, \tau_{2}, \ldots, \tau_{M}\right)\left(0=\tau_{0} \leq \tau_{1} \leq \tau_{2} \leq \cdots \leq \tau_{M}, M \geq 1\right.$ is a given natural number) with no refraction. The stochastic process $Z_{t}(t \geq 0)$ is the geometric Brownian motion governed by the Itô SDE

$$
\mathrm{d} Z_{t}=Z_{t}\left(r(t) \mathrm{d} t+\sigma(t) \mathrm{d} B_{t}\right), \quad t>0
$$

with $(r(t), \sigma(t))=\left(r_{i}, \sigma_{i}\right)$ for $\tau_{i-1}<t \leq \tau_{i}$ where $r_{i}, \sigma_{i}>0,2 r_{i}>\sigma_{i}^{2}$ are given constants. We put $\Phi_{M+1}(z)=z^{1-\alpha} /(1-\alpha)$ for the sake of brevity. The stopping times are chosen to maximize the performance index

$$
J_{\tau}(z)=\mathrm{E}\left[\sum_{i=1}^{M} \eta_{i} \int_{\tau_{i-1}}^{\tau_{i}} \frac{q_{i}}{1-\beta_{i}} Z_{s}^{1-\beta_{i}} e^{-\delta_{i}\left(s-\tau_{i-1}\right)} \mathrm{d} s+\eta_{M+1} \Phi_{M+1}\left(Z_{\tau_{M}}\right) \mid Z_{0}=z\right]
$$

(c) The Author(s) 2018. This article is distributed under the terms of the Creative Commons Attribution 4.0 International License (http://creativecommons.org/licenses/by/4.0/), which permits unrestricted use, distribution, and reproduction in any medium, provided you give appropriate credit to the original author(s) and the source, provide a link to the Creative Commons license, and indicate if changes were made. 
with

$$
\eta_{i}=e^{-\sum_{j=1}^{i-1} \delta_{j}\left(\tau_{j}-\tau_{j-1}\right)}
$$

where $\mathrm{E}$ is the expectation operator, $\delta_{i}>0, q_{i}>0$, and $0<\alpha<\beta_{M}<\beta_{M-1}<\cdots<\beta_{1}<1$ are given constants and $z \geq 0$ is the initial condition of $Z_{t}$. We show that an application of the dynamic programming principle reduces the present optimal stopping problem to a series of variational inequalities (VIs). The VIs, and consequently the present multiple optimal stopping problem, turn out to be exactly solvable. Its implications in an ecological problem are briefly discussed as well.

The main difference between the present model and the existing models [8-11] is that the former has an ecological background, while the latter have the financial backgrounds. In addition, the performance indices to be maximized or minimized have different functional forms with each other. The resulting VIs have different forms as well. The main contribution of this paper is the derivation of an exact solution to the cascading system of VIs and its ecological implications.

\section{Variational inequalities}

The value function $\Phi=\Phi(z)$ is defined with the performance index $J_{\tau}(z)$ as

$$
\Phi(z)=\sup _{\tau} J_{\tau}(z)
$$

By the strong Markov property of the process $Z_{t},(4)$ is rewritten as

$$
\Phi(z)=\sup _{\tau_{1}, \tau_{2}, \ldots, \tau_{M-1}} \mathrm{E}\left[\sum_{i=1}^{M-1} \eta_{i} \int_{\tau_{i-1}}^{\tau_{i}} \frac{q_{i}}{1-\beta_{i}} Z_{s}^{1-\beta_{i}} e^{-\delta_{i}\left(s-\tau_{i-1}\right)} \mathrm{d} s+\eta_{M} \Phi_{M}\left(Z_{\tau_{M-1}}\right) \mid Z_{0}=z\right]
$$

where

$$
\begin{aligned}
\Phi_{M}(y)= & \sup _{\tau_{M-1} \leq \tau_{M}} \mathrm{E}\left[\int_{\tau_{M-1}}^{\tau_{M}} \frac{q_{M}}{1-\beta_{M}} Z_{s}^{1-\beta_{M}} e^{-\delta_{M}\left(s-\tau_{M-1}\right)} \mathrm{d} s+\Phi_{M+1}\left(Z_{\tau_{M}}\right) e^{-\delta_{M}\left(\tau_{M}-\tau_{M-1}\right)} \mid\right. \\
& \left.Z_{\tau_{M-1}}=y\right] .
\end{aligned}
$$

Similarly, introduce the functions $\Phi_{i}(y)$ for $y \geq 0$ recursively as

$$
\begin{aligned}
\Phi_{i}(y) & =\sup _{\tau_{i-1} \leq \tau_{i}} \mathrm{E}\left[\int_{\tau_{i-1}}^{\tau_{i}} \frac{q_{i}}{1-\beta_{i}} Z_{s}^{1-\beta_{i}} e^{-\delta_{i}\left(s-\tau_{i-1}\right)} \mathrm{d} s+\Phi_{i+1}\left(Z_{\tau_{i}}\right) e^{-\delta_{i}\left(\tau_{i}-\tau_{i-1}\right)} \mid Z_{\tau_{i-1}}=y\right], \\
1 & \leq i \leq M .
\end{aligned}
$$

The recursive equations (6) and (7) are later utilized to show that the present multiple optimal stopping problem results in a cascading system of VIs that can be solved in a cascading manner from $i=M$ to $i=1$. The value function $\Phi$ is then obtained: $\Phi(z)=\Phi_{1}(z)$. Assume $\Phi_{i} \in C^{1}(0,+\infty) \cap C^{0}([0,+\infty))$ for $1 \leq i \leq M$ and is twice continuously differentiable almost everywhere in $\mathbb{R}^{+}$, which is true under certain assumptions. The degenerate 
elliptic operator $L_{i}$ is defined as

$$
L_{i} \Psi=\delta_{i} \Psi-r_{i} z \frac{\mathrm{d} \Psi}{\mathrm{d} z}-\frac{1}{2} \sigma_{i}^{2} z^{2} \frac{\mathrm{d}^{2} \Psi}{\mathrm{d} z^{2}}
$$

for generic sufficiently smooth $\Psi=\Psi(z)$. Then Theorem 10.4.1 [12] shows that $\Phi_{i}(1 \leq$ $i \leq M$ ) solves the VI

$$
\min \left\{L_{i} \Phi_{i}-\frac{q_{i}}{1-\beta_{i}} z^{1-\beta_{i}}, \Phi_{i}-\Phi_{i+1}\right\}=0, \quad z>0 .
$$

The VI (9) is subject to the boundary condition $\Phi_{i}(0)=0$.

\section{Main result}

Define $k_{i}$ and $\lambda_{i}(1 \leq i \leq M)$ as

$$
k_{i}=\frac{1}{\sigma_{i}^{2}}\left[-\left(r_{i}-\frac{\sigma_{i}^{2}}{2}\right)+\sqrt{\left(r_{i}-\frac{\sigma_{i}^{2}}{2}\right)^{2}+2 \delta_{i} \sigma_{i}^{2}}\right]
$$

and

$$
\lambda_{i}=\delta_{i}-\left(1-\beta_{i}\right) r_{i}+\frac{1}{2} \sigma_{i}^{2} \beta_{i}\left(1-\beta_{i}\right),
$$

respectively. Theorem 1 is the main result of this paper, which shows that the VIs of the present multiple optimal stopping problem are exactly solvable.

Theorem 1 Assume $\delta_{i}>r_{i}$ and $\lambda_{i}>0$ for $1 \leq i \leq M$, and $k_{i}>k_{i+1}$ for $1 \leq i \leq M-1$ when $M \geq 2$. Then there is a sequence $0<q_{1}<q_{2}<\cdots<q_{M}<+\infty$ such that $\Phi_{i} \in C^{1}(0,+\infty) \cap$ $C^{0}([0,+\infty))(1 \leq i \leq M)$ is uniquely expressed as

$$
\Phi_{i}(z)= \begin{cases}A_{i} z^{k_{i}}+B_{i} z^{1-\beta_{i}} & \left(0 \leq z \leq \bar{z}_{i}\right), \\ A_{i+1} z^{k_{i+1}}+B_{i+1} z^{1-\beta_{i+1}} & \left(\bar{z}_{i}<z<\bar{z}_{i+1}\right),\end{cases}
$$

with some sequence $0<\bar{z}_{1}<\bar{z}_{2}<\cdots<\bar{z}_{M}<\bar{z}_{M+1}=+\infty$ and $A_{i}, B_{i}>0$ for $1 \leq i \leq M$. Here, $A_{M+1}=0, B_{M+1}=1 /(1-\alpha)$, and $\beta_{M+1}=\alpha$. In addition, $\Phi_{i}=\Phi_{i}(z), z>0$ is twice continuously differentiable except at the $M-i+1$ points $\bar{z}_{i}, \bar{z}_{i+1}, \ldots, \bar{z}_{M}$.

Proof of Theorem 1 By (10), it is straightforward to check that the assumption $\delta_{i}>r_{i}$ leads to $k_{i}>1$. General solutions $\Psi \in C^{2}(0,+\infty) \cap C^{0}([0,+\infty))$ to the problem

$$
L_{i} \Psi=0, \quad z>0, \quad \Psi(0)=0
$$

are expressed with a real constant $c$ as $\Psi(z)=c z^{k_{i}}$. For $i=M$, we have a candidate of the solution of the form (12) with $B_{M}=q_{M} /\left[\lambda_{M}\left(1-\beta_{M}\right)\right]$ where $B_{M} z^{1-\beta_{M}}$ is the particular solution to $L_{M} \Phi_{M}-q_{M} z^{1-\beta_{M}} /\left(1-\beta_{M}\right)=0, z>0$. There are two unknowns, $\bar{z}_{M}$ and $A_{M}$, which are determined from the smooth-pasting condition [13] at $z=\bar{z}_{M} \geq 0$ :

$$
\begin{aligned}
& A_{M} z^{k_{M}}+B_{M} z^{1-\beta_{M}}=\frac{1}{1-\beta_{M+1}} z^{1-\beta_{M+1},} \\
& A_{M} k_{M} z^{k^{-1}}+B_{M}\left(1-\beta_{M}\right) z^{-\beta_{M}}=z^{-\beta_{M+1}}, \quad z=\bar{z}_{M} .
\end{aligned}
$$


The second equation of (14) excludes the case with $\bar{z}_{M}=0$. Equation (14) is uniquely solved as

$$
\begin{aligned}
& A_{M}=\frac{\beta_{M}-\beta_{M+1}}{1-\beta_{M}} \frac{\left(\bar{z}_{M}\right)^{-\left(k_{M}-1+\beta_{M+1}\right)}}{k_{M}-1+\beta_{M}}>0, \\
& \bar{z}_{M}=\left[\frac{1-\beta_{M+1}}{1-\beta_{M}} \frac{k_{M}-1+\beta_{M}}{k_{M}-1+\beta_{M+1}} \frac{q_{M}}{\lambda_{M}}\right]^{\frac{1}{\beta_{M}-\beta_{M+1}}}>0 .
\end{aligned}
$$

Uniqueness of the solution $\Phi_{M}$ to VI (9) in a viscosity sense follows from an infinite horizon counterpart of Theorem 3.1 [14]. Regularity conditions $\Phi_{M} \in C^{1}(0,+\infty) \cap C^{0}([0,+\infty))$ and $\Phi_{M} \in C^{2}\left(0, \bar{z}_{M}\right) \cap C^{2}\left(\bar{z}_{M},+\infty\right)$ directly follow from the form of $\Phi_{M}$. Hence, $\Phi_{M}=$ $\Phi_{M}(z)$ is twice continuously differentiable almost everywhere for $z>0$.

The discussion above can be continued for $1 \leq i \leq M-1$ with $M \geq 2$. The proof in what follows is based on a recursive argument. We firstly assume $0<\bar{z}_{1}<\bar{z}_{2}<\cdots<\bar{z}_{M}<+\infty$ and later show that this assumption is satisfied by appropriately choosing the sequence $0<q_{1}<q_{2}<\cdots<q_{M}<+\infty$. Assume that the statement of the theorem is true for all $j$ such that $i+1 \leq j \leq M$. Then, from VI (9), we find that the candidate of its solution is expressed as (12) with $B_{i}=q_{i} /\left[\lambda_{i}\left(1-\beta_{i}\right)\right]$ where $B_{i} z^{1-\beta_{i}}$ is the particular solution to $L_{i} \Phi_{i}-q_{i} z^{1-\beta_{i}} /(1-$ $\left.\beta_{i}\right)=0, z>0$. As in the case for $i=M$, there are two unknowns $\bar{z}_{i}$ and $A_{i}$ in (12). They are determined from the smooth-pasting condition at $z=\bar{z}_{i} \geq 0$ :

$$
\begin{aligned}
& A_{i} z^{k_{i}}+B_{i} z^{1-\beta_{i}}=A_{i+1} z^{k_{i+1}}+B_{i+1} z^{1-\beta_{i+1}}, \\
& A_{i} k_{i} z^{k_{i}-1}+B_{i}\left(1-\beta_{i}\right) z^{-\beta_{i}}=A_{i+1} k_{i+1} z^{k_{i+1}-1}+B_{i+1}\left(1-\beta_{i+1}\right) z^{-\beta_{i+1}}, \quad z=\bar{z}_{i}<\bar{z}_{i+1} .
\end{aligned}
$$

The second equation of (14) excludes the case with $\bar{z}_{i}=0$. A remarkable difference between the cases with $i<M$ and $i=M$ is that $\bar{z}_{i}$ and $A_{i}$ cannot be expressed explicitly in general in the former case. Fortunately, they are uniquely found from (16) as shown below. Combining the two equations of (16) leads to the equation to be solved by $\bar{z}_{i}$ :

$$
B_{i}\left(k_{i}-1+\beta_{i}\right)=f_{i}(z)
$$

where $f_{i}(z)$ for $z \geq 0$ is the polynomial

$$
f_{i}(z)=A_{i+1}\left(k_{i}-k_{i+1}\right) z^{k_{i+1}-1+\beta_{i}}+B_{i+1}\left(k_{i}-1+\beta_{i}\right) z^{\beta_{i}-\beta_{i+1}} .
$$

The left-hand side of (17) and all the coefficients and powers appearing in $f_{i}$ are positive by the assumption of the theorem. In addition, $f_{i}(z)$ is monotonically increasing with respect to $z>0, \lim _{z \rightarrow+\infty} f_{i}(z) \rightarrow+\infty$, and $f_{i}(0)=0$. Therefore, Eq. (17) admits a unique positive solution by the classical intermediate theorem: namely, $\bar{z}_{i}>0$. Substituting this $\bar{z}_{i}$ into (16) uniquely yields $A_{i}$. A useful result on $f_{i}$ is that

$$
z<\bar{z}_{i}\left(z>\bar{z}_{i}\right) \text { when } \quad B_{i}\left(k_{i}-1+\beta_{i}\right)>f_{i}(z) \quad\left(B_{i}\left(k_{i}-1+\beta_{i}\right)<f_{i}(z)\right)
$$

due to its monotonicity. The sign of $A_{i}$ is positive as shown in what follows. Combining the two equations of (16) yields

$$
A_{i}\left(k_{i}-k_{i+1}\right) z^{k_{i}}=g_{i}(z), \quad z=\bar{z}_{i}
$$


where $g_{i}(z)$ for $z \geq 0$ is defined as

$$
g_{i}(z)=B_{i}\left(k_{i+1}-1+\beta_{i}\right) z^{1-\beta_{i}}-B_{i+1}\left(k_{i+1}-1+\beta_{i+1}\right) z^{1-\beta_{i+1}} .
$$

By (20) and the assumptions of the theorem, $A_{i}>0$ if $g_{i}\left(\bar{z}_{i}\right)>0$. Define $\tilde{z}_{i}>0$ as

$$
\tilde{z}_{i}=\left[\frac{B_{i}\left(k_{i+1}-1+\beta_{i}\right)}{B_{i+1}\left(k_{i+1}-1+\beta_{i+1}\right)}\right]^{\frac{1}{\beta_{i}-\beta_{i+1}}}
$$

which is the unique solution to $g_{i}(z)=0$ for $z>0$. By the functional form of $g_{i}, g_{i}(z)<0$ for $z>\tilde{z}_{i}$. Thus, $A_{i}>0$ if $\bar{z}_{i}<\tilde{z}_{i}$.

By $(19), \bar{z}_{i}<\tilde{z}_{i}$ when $B_{i}\left(k_{i}-1+\beta_{i}\right)<f_{i}\left(\tilde{z}_{i}\right)$. The quantity $f_{i}\left(\tilde{z}_{i}\right)$ is calculated as

$$
\begin{aligned}
f_{i}\left(\tilde{z}_{i}\right) & =A_{i+1}\left(k_{i}-k_{i+1}\right) \tilde{z}_{i}^{k_{i+1}-1+\beta_{i}}+B_{i+1}\left(k_{i}-1+\beta_{i}\right) \tilde{z}_{i}^{\beta_{i}-\beta_{i+1}} \\
& =A_{i+1}\left(k_{i}-k_{i+1}\right) \tilde{z}_{i}^{k_{i+1}-1+\beta_{i}}+B_{i} \frac{\left(k_{i}-1+\beta_{i+1}\right)\left(k_{i+1}-1+\beta_{i}\right)}{k_{i+1}-1+\beta_{i+1}} .
\end{aligned}
$$

Define $l_{i}$ as

$$
l_{i}=k_{i}-1+\beta_{i}-\frac{\left(k_{i}-1+\beta_{i+1}\right)\left(k_{i+1}-1+\beta_{i}\right)}{k_{i+1}-1+\beta_{i+1}} .
$$

By (24), the right-hand side of (23) is positive. A straightforward calculation shows

$$
\begin{aligned}
\left(k_{i+1}-1+\beta_{i+1}\right) l_{i} & =\left(k_{i}-1+\beta_{i}\right)\left(k_{i+1}-1+\beta_{i+1}\right)-\left(k_{i}-1+\beta_{i+1}\right)\left(k_{i+1}-1+\beta_{i}\right) \\
& =\left(k_{i+1}-k_{i}\right)\left(\beta_{i}-\beta_{i+1}\right) \\
& <0,
\end{aligned}
$$

namely, the desired inequality

$$
l_{i}<0
$$

since $k_{i}>k_{i+1}$ and $\beta_{i}>\beta_{i+1}$. The inequality $B_{i}\left(k_{i}-1+\beta_{i}\right)<f_{i}\left(\tilde{z}_{i}\right)$ follows from (26). Therefore, we have $\bar{z}_{i}<\tilde{z}_{i}$ and thus $A_{i}>0$.

Uniqueness of the solution $\Phi_{i} \in C^{1}(0,+\infty) \cap C^{0}([0,+\infty))$ is then proven as follows. In addition, $\Phi_{i}=\Phi_{i}(z)$ is identified with $\Phi_{i+1}=\Phi_{i+1}(z)$ for $z>\bar{z}_{i}$ by the construction, meaning that $\Phi_{i}$ is twice continuously differentiable except at the $M-i+1$ points $\bar{z}_{i}, \bar{z}_{i+1}, \ldots, \bar{z}_{M}$. Furthermore, uniqueness of the solution $\Phi_{i}$ to VI (9) in a viscosity sense follows from an infinite horizon counterpart of Theorem 3.1 [14]. Therefore, by the induction, it is shown that the statement of the theorem is true if we can construct a sequence $0<\bar{z}_{1}<\bar{z}_{2}<\cdots<$ $\bar{z}_{M}<+\infty$. This issue is not encountered for $M=1$ where the problem involves a single optimal stopping time, since we have $0<\bar{z}_{1}<+\infty$.

Assume $M \geq 2$. By the second equation of (15), $\bar{z}_{M}$ can be seen as an increasing power function of $q_{M}$. In addition, $B_{M}$ is increasing with respect to $q_{M}$. Therefore, for a fixed $q_{M-1}$, it is possible to choose a sufficiently large $q_{M}$ such that

$$
B_{i}\left(k_{i}-1+\beta_{i}\right)<f_{i}\left(\bar{z}_{M}\right)=A_{i+1}\left(k_{i}-k_{i+1}\right) \bar{z}_{M}^{k_{i+1}-1+\beta_{i}}+B_{i+1}\left(k_{i}-1+\beta_{i}\right) \bar{z}_{M}^{\beta_{i}-\beta_{i+1}} .
$$


This inequality means that there exist $\bar{z}_{M-1}$ and $\bar{z}_{M}$ with $0<\bar{z}_{M-1}<\bar{z}_{M}<+\infty$ by appropriately choosing $q_{M-1}$ and $q_{M}$. As a next step, assume $M=3$ for the sake of simplicity. The argument below can straightforwardly be extended to $M \geq 3$. Assume that the condition $0<\bar{z}_{2}<\bar{z}_{3}<+\infty$ is satisfied. The explicit expression of $\bar{z}_{1}$ is not available, but it satisfies

$$
\lim _{q_{1} \rightarrow+0} \bar{z}_{1}=0
$$

Actually, (17) and (18) with $i=1$ show that the left-hand side of (17) is increasing with respect to $q_{1}$, while the right-hand side of (17) is independent of $q_{1}$. Therefore, we can choose a sufficiently small $q_{1}>0$ such that $B_{1}\left(k_{1}-1+\beta_{1}\right)<f_{1}\left(\bar{z}_{2}\right)$; namely, $0<\bar{z}_{1}<\bar{z}_{2}$. We then have $0<\bar{z}_{1}<\bar{z}_{2}<\bar{z}_{3}<+\infty$. The proof for $M \geq 3$ is essentially the same.

The following proposition is proven in an essentially similar way with Theorem 1.

Proposition 2 Replace " $k_{i}>k_{i+1}$ for $1 \leq i \leq M-1$ when $M \geq 2$ " by " $k_{i}=k_{i+1}$ for $1 \leq i \leq$ $M-1$ when $M \geq 2$ " in Theorem 1 . Then we have $\Phi_{i}(1 \leq i \leq M)$ of the form (12) where

$$
A_{i}=\frac{\beta_{i}-\beta_{i+1}}{1-\beta_{i}} \frac{\left(\bar{z}_{i}\right)^{-\left(k_{i}-1+\beta_{i+1}\right)}}{k_{i}-1+\beta_{i}}>0 \quad \text { and } \quad \bar{z}_{i}=\left[\frac{1-\beta_{i+1}}{1-\beta_{i}} \frac{k_{i}-1+\beta_{i+1}}{k_{i}-1+\beta_{i}} \frac{\lambda_{i+1} q_{i}}{\lambda_{i} q_{i+1}}\right]^{\frac{1}{\beta_{i}-\beta_{i+1}}}
$$

The second equation of (29) shows that $\bar{z}_{i}$ is expressed as a monotonically increasing and unbounded function of $q_{i} / q_{i+1}$, implying that $\bar{z}_{M-1}<\bar{z}_{M}$ if $q_{M}$ is sufficiently larger than $q_{M-1}$. Similarly, we have $\bar{z}_{M-2}<\bar{z}_{M-1}$ if $q_{M-1}$ is sufficiently larger than $q_{M-2}$. We can choose larger $q_{M}$ if necessary. Since $M$ is bounded, we can construct a sequence $0<q_{1}<q_{2}<\cdots<$ $q_{M}<+\infty$ such that $0<\bar{z}_{1}<\bar{z}_{2}<\cdots<\bar{z}_{M}<+\infty$.

An immediate consequence of Theorem 1 and Proposition 2 is the next proposition.

Proposition $3 \Phi_{1}$ is the value function $\Phi$ under the assumption of Theorem 1 or that of Proposition 2.

Remark 4 A numerical example of Theorem 1 is provided. Set the following parameter values: $\delta_{1}=6.5, \delta_{2}=4, r_{1}=3, r_{2}=2, \sigma_{1}=0.5, \sigma_{2}=0.2, \beta_{1}=0.8, \beta_{2}=0.5, \beta_{3}=0.1, q_{1}=0.4$, and $q_{2}=0.9$. In this case, the growth rate $r_{i}$ of the animal population increases while its fluctuation $\sigma_{i}$ decreases as $i$ increases, which is an ecologically reasonable situation. Based on these parameter values, we have $k_{1}=2.074>k_{2}=1.981, \lambda_{1}=5.920$, and $\lambda_{2}=3.005$. These given and calculated constants comply with the assumption of Theorem 1 . Furthermore, we have $A_{1}=1.393, A_{2}=1.224, B_{1}=0.338, B_{2}=0.599, B_{3}=1.111, \bar{z}_{1}=0.145$, and $\bar{z}_{2}=0.469$. The obtained results satisfy $0<\bar{z}_{1}<\bar{z}_{2}$ and $A_{1}, A_{2}>0$, which comply with the results of Theorem 1.

Remark 5 Each optimal $\tau_{i}$ is denoted $\tau_{i}^{*}$. Under the assumption of Theorem 1 or that of Proposition 2, the optimal stopping time $\tau_{i}^{*}$ is expressed as

$$
\tau_{i}^{*}=\inf \left\{\tau \mid \tau>\tau_{i-1}^{*}, Z_{\tau}=\bar{z}_{i}\right\} \quad \text { for } 1 \leq i \leq M, \tau_{0}^{*}=0
$$

Remark 6 The strong Markov property of geometric Brownian motions [15] gives the following formulas for the statistical moments of the largest stopping time $\tau_{M}$. For example, 
Table 1 Meaning of the variables and parameters of the present multiple optimal stopping problem in analyzing fish migration

\begin{tabular}{ll}
\hline Parameter & Meaning \\
\hline$r_{i}$ & Deterministic growth rate of the population biomass in the habitat $H_{i}$ \\
$\sigma_{i}$ & Stochasticity involved in the growth of the population biomass in the habitat $H_{i}$ \\
$\delta_{i}$ & Discount factor of the cumulative profit in the habitat $H_{i}$ \\
$q_{i}$ & Quality of the habitat $H_{i}$ \\
$\beta_{i}$ & Sensitivity of the profit gained in the habitat $H_{i}$ on the population biomass \\
\hline
\end{tabular}

a straightforward calculation gives

$$
\mathrm{E}\left[\tau_{M}^{*}\right]=\mathrm{E}\left[\sum_{i=1}^{M}\left(\tau_{i}^{*}-\tau_{i-1}^{*}\right)\right]=\sum_{i=1}^{M} \mathrm{E}\left[\tau_{i}^{*}-\tau_{i-1}^{*} \mid Z_{\tau_{i-1}^{*}}\right] .
$$

Since $Z_{\tau_{i}^{*}}=\bar{z}_{i}(1 \leq i \leq M)$ and $Z_{0}=z>0$,

$$
\mathrm{E}\left[\tau_{M}^{*}\right]=\sum_{i=1}^{M} \frac{2}{2 r_{i}-\sigma_{i}^{2}} \ln \left(\frac{\bar{z}_{i}}{\bar{z}_{i-1}}\right),
$$

where the notation $\bar{z}_{0}=z$ is employed for the sake of simplicity. Similarly, the variance of $\tau_{M}$ is found as

$$
\operatorname{Var}\left[\tau_{M}^{*}\right]=\sum_{i=1}^{M} \operatorname{Var}\left[\tau_{i}^{*}-\tau_{i-1}^{*} \mid Z_{\tau_{i-1}^{*}}\right]=\sum_{i=1}^{M} \sigma_{i}^{2}\left(\frac{2}{2 r_{i}-\sigma_{i}^{2}}\right)^{3} \ln \left(\frac{\bar{z}_{i}}{\bar{z}_{i-1}}\right) .
$$

The present multiple optimal stopping problem is a simple theoretical model for migration of animals, migratory fishes in particular [16]. A single optimal stopping problem for analyzing animal migration between two habitats has been discussed in Yoshioka and Yaegashi [7] from a numerical viewpoint. Assume that there are $M+1$ habitats, which are denoted $H_{0}, H_{1}, \ldots, H_{M}$ where $H_{0}$ is the initial habitat and $H_{M}$ is the final habitat: the goal of the migration. The stochastic process $Z_{t}$ represents the biomass of an animal population at the time $t$. The stopping time $\tau_{i}$ represents the time to move from $H_{i-1}$ to $H_{i}$. The objective of the animal population is to choose the sequence of stopping times $\tau_{i}(1 \leq i \leq M)$, so that the sum of the cumulative profit gain in each habitat $H_{i}(0 \leq i \leq M-1)$ and the terminal wealth gained at the goal of migration $H_{M}$, namely the performance index $J_{\tau}$ in (2), is maximized.

An example is the migratory fish Plecoglossus altivelis (P. altivelis, Ayu) having an annual life cycle that migrates between a river and the seas [17]. The present mathematical model can be applied to modelling one-generation life history of the fish with $M=2$. In each autumn, the adults spawn eggs in downstream reaches of a river in which they live and die soon afterward. Hatched larvae descend to coastal areas of a downstream water body of the river: the sea or an estuary $\left(H_{0}\right)$. In the coming spring, grown fishes ascend into the midstream of the river to mature $\left(H_{1}\right)$ until the coming autumn. In the autumn, the fishes descend to the downstream reach of the river where they can spawn $\left(H_{2}\right)$. Table 1 summarizes the meaning of the model parameters in the above-mentioned problem.

The assumptions $\delta_{i}>r_{i}$ and $\lambda_{i}>0$ for $1 \leq i \leq M$ and $k_{i}>k_{i+1}$ for $1 \leq i \leq M-1$ when $M \geq 2$ actually have ecological meanings for the animal migration problems. The conditions $\delta_{i}>r_{i}$ and $\lambda_{i}>0$ can be restated as that $\delta_{i}>0$ is sufficiently large, implying that the 
habitat quality degrades as the time elapses. This is in accordance with the fact that animal migration is often driven by seasonal changes of habitat quality. The remaining condition $k_{i}>k_{i+1}$ is satisfied if $\delta_{i}$ is sufficiently larger than $\delta_{i+1}$. For the animal migration, this condition to the situation where degradation of the habitat quality is critical for the earlier period of the animal life history.

\section{Conclusions}

This paper focused on a solvable multiple optimal stopping problem related to animal migration. An extension of the present problem is to consider a refraction $\tau_{i+1}-\tau_{i} \geq \mu_{i}>0$, which leads to a different system of VIs, and consequently different value functions and optimal stopping criteria. Solvability of the problem with a refraction is currently under investigation for more realistic mathematical modelling of animal migration. There exist recent studies on optimization models of ecological and biological systems involving delays [18-20]. These systems are clearly more complicated than the system focused on in this paper. To examine the applicability of the present methodology, to extend these models will be a quite interesting topic. Applicability of the present formalism to real animal migration, which is based on a mixed control problem like those in Koo et al. [21] and Lee and Shin [22], is also currently in progress.

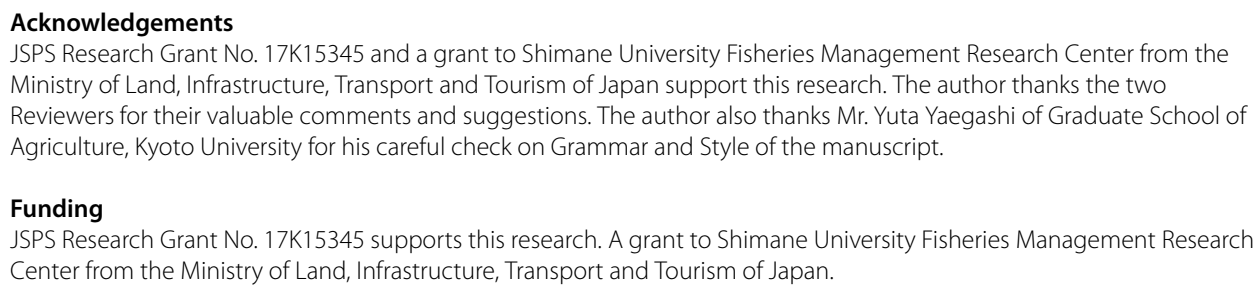

HY carried out mathematical analysis and wrote the manuscript. Author read and approved the final manuscript.

\section{Publisher's Note}

Springer Nature remains neutral with regard to jurisdictional claims in published maps and institutional affiliations.

Received: 17 October 2017 Accepted: 2 May 2018 Published online: 08 May 2018

\section{References}

1. Jacka, S.L.: Optimal stopping and the American put. Math. Finance 1, 1-14 (1991). https://doi.org/10.1111/j.1467-9965.1991.tb00007.x

2. Zhu, S.P., Le, N.T., Chen, W., Lu, X.: Pricing Parisian down-and-in options. Appl. Math. Lett. 43, 19-24 (2015) https://doi.org/10.1016/j.aml.2014.10.019

3. Pindyck, R.S.: Optimal timing problems in environmental economics. J. Econ. Dyn. Control 26, 1677-1697 (2002). https://doi.org/10.1016/S0165-1889(01)00090-2

4. Framstad, N.C., Strand, J.: Energy intensive infrastructure investments with retrofits in continuous time: effects of uncertainty on energy use and carbon emissions. Resour. Energy Econ. 41, 1-18 (2015). https://doi.org/10.1016/j.reseneeco.2015.03.003

5. Reed, W.J.: The decision to conserve or harvest old-growth forest. Ecol. Econ. 8, 45-69 (1993). https://doi.org/10.1016/0921-8009(93)90030-A

6. Sims, C., Finnoff, D., Shogren, J.F.: Bioeconomics of invasive species: using real options theory to integrate ecology, economics, and risk management. Food Secur. 8, 61-70 (2016). https://doi.org/10.1007/s12571-015-0530-1 
7. Yoshioka, Y., Yaegashi, Y.: Numerical simulation of animal migration via a nonlinear degenerate elliptic free boundary problem. In: Proceedings of the 36th JSST Annual International Conference on Simulation Technology. Proceedings, pp. 174-177 (2017)

8. Carmona, R., Dayanik, S.: Optimal multiple stopping of linear diffusions. Math. Oper. Res. 33, 446-460 (2008). https://doi.org/10.1287/moor.1070.0301

9. Carmona, R., Touzi, N.: Optimal multiple stopping and valuation of swing options. Math. Finance 18, 239-268 (2008). https://doi.org/10.1111/j.1467-9965.2007.00331.x

10. Cai, N., Sun, L.: Valuation of stock loans with jump risk. J. Econ. Dyn. Control 40, 213-241 (2014). https://doi.org/10.1016/j.jedc.2014.01.004

11. Dai, M., Kwok, Y.K.: Optimal multiple stopping models of reload options and shout options. J. Econ. Dyn. Control 32, 2269-2290 (2008). https://doi.org/10.1016/j.jedc.2007.10.002

12. Øksendal, B.: Stochastic Differential Equations. Springer, Berlin (2003)

13. Dixit, A.K., Pindyck, R.S.: Investment Under Uncertainty. Princeton University Press, Princeton (1994)

14. Reikvam, K.: Viscosity solutions of optimal stopping problems. Stoch. Stoch. Rep. 62, 285-301 (1998). https://doi.org/10.1080/17442509808834137

15. Zhang, L., Du, Z:: On the reflected geometric Brownian motion with two barriers. Intell. Inform. Manag. 2, 295-298 (2010). https://doi.org/10.4236/iim.2010.23034

16. Zydlewski, J., Wilkie, M.P.: Freshwater to seawater transitions in migratory fishes. Fish Physiol. 32, 253-326 (2013). https://doi.org/10.1016/B978-0-12-396951-4.00006-2

17. Yaegashi, Y., Yoshioka, H., Unami, K., Fujihara, M.: An optimal management strategy for stochastic population dynamics of released Plecoglossus altivelis in rivers. Int. J. Model. Sim. Sci. Comput. 8, 1750039 (2017). https://doi.org/10.1142/S1793962317500398

18. Liu, L., Meng, X.: Optimal harvesting control and dynamics of two-species stochastic model with delays. Adv. Differ. Equ. 2017, 18 (2017). https://doi.org/10.1186/s13662-017-1077-6

19. Feng, T., Meng, X., Liu, L., Gao, S.: Application of inequalities technique to dynamics analysis of a stochastic eco-epidemiology model. J. Inequal. Appl. 2016, 327 (2016). https://doi.org/10.1186/s13660-016-1265-z

20. Leng, X., Feng, T., Meng, X.: Stochastic inequalities and applications to dynamics analysis of a novel SIVS epidemic model with jumps. J. Inequal. Appl. 2017, 138 (2017). https://doi.org/10.1186/s13660-017-1418-8

21. Koo, J.L., Koo, B.L., Shin, Y.H.: An optimal investment, consumption, leisure, and voluntary retirement problem with Cobb-Douglas utility: dynamic programming approaches. Appl. Math. Lett. 26, 481-486 (2013). https://doi.org/10.1016/j.aml.2012.11.012

22. Lee, H.S., Shin, Y.H.: An optimal investment, consumption-leisure and voluntary retirement choice problem with subsistence consumption constraints. Jpn. J. Ind. Appl. Math. 33, 297-320 (2016). https://doi.org/10.1007/s13160-016-0215-y

\section{Submit your manuscript to a SpringerOpen ${ }^{\circ}$ journal and benefit from:}

- Convenient online submission

- Rigorous peer review

Open access: articles freely available online

- High visibility within the field

Retaining the copyright to your article

Submit your next manuscript at $\boldsymbol{~ s p r i n g e r o p e n . c o m ~}$ 